\title{
Use of graphics software in radiative heat transfer simulation
}

\author{
K. Domke \\ Institute of Industrial Electrical Engineering, \\ Poznan University of Technology, Poland
}

\begin{abstract}
A base equation of radiative heat transfer, along with typical calculation methods, has been presented. Goals and base equation of computer graphics programs have been described. A quick analysis of graphics programs has been made, and their functions have been compared with the functions of other programs for radiative heat transfer modeling. Conditions that should be fulfilled by graphics programs in order to be used for modeling radiative transfer have been given. Formulas transforming heat quantities to radiant quantities, as used for Dirichlet's and Neumann's boundary conditions, have been presented. Keywords: modeling of radiative heat transfer, graphics software.
\end{abstract}

\section{Introduction}

Radiative heat transfer is one of three basic ways of heat transfer. It is a common phenomenon, consisting in heat energy (power) transfer through electromagnetic (infrared) radiation, mainly with a wavelength of $\lambda \in(0,78-1000) \mu \mathrm{m}$, between nontransparent (or translucent) surfaces of temperature above $0 \mathrm{~K}$. Even in temperature conditions of $(0-50)^{\circ} \mathrm{C}$ range, for non-vacuum systems, the share of this method of heat transfer is ca $(15-20) \%$ of the total heat transfer, and increases significantly in higher temperatures. It is also the only method of heat transfer in vacuum systems. In many electrotechnical devices determining temperature fields related to normal work and operation of these devices is a basic condition for verifying the correctness of their construction and it determines their admissible loads. Hence the significance of methods allowing one to calculate temperature distributions and power fluxes related to heat transfer, including radiative heat transfer. Omitting radiative heat transfer in 
general heat calculations causes at least (15-20)\% of errors while determining values of heat quantities.

\section{Radiative heat transfer, principles and base equation, calculation methods}

Each body of temperature above $0 \mathrm{~K}$ emits electromagnetic radiation into hemisphere. In case of a black body (indices "bb"), it is described by Planck's law $[6,8]$ :

$$
m_{b b, \lambda}=c_{1} \lambda^{-5}\left(\frac{c_{2}}{e^{\lambda T}}-1\right)^{-1}
$$

which shows the dependence of monochromatic radiance $m_{b b, \lambda}$ (or, in other words, monochromatic power surface density) of a black body from its temperature $T$. Radiance of a real body is described by the following dependence

$$
m_{\lambda}=\varepsilon_{\lambda} m_{b b, \lambda}
$$

It is monochromatic radiance density of primary radiation (measured in $\left.\left[\mathrm{W} /\left(\mathrm{m}^{2} \mathrm{sr} \mu \mathrm{m}\right)\right]\right)$, emitted from the surface of temperature $T$ and emissivity $\varepsilon$.

Radiation should usually be considered while taking into account the direction into which it propagates. In such a case a notion of monochromatic radiance density is introduced, defined as monochromatic radiance density $l$ measured in a solid angle $\mathrm{d} \omega$ emitted from a perpendicular surface $S_{p}$

$$
l_{\lambda}=\frac{d m_{\lambda}}{d \omega}
$$

or for total quantities which do not take into consideration the dependence on wavelength $\lambda$

$$
L=\frac{d M}{d \omega}
$$

which may also be presented by this formula $[1,2,3,6]$

$$
L=\frac{d^{2} \Phi}{d \omega \cdot d S_{p}}=\frac{d^{2} \Phi}{d \omega \cdot d S \cdot \cos \eta}
$$

where $L$ is radiance, measured in $\left[\mathrm{W} /\left(\mathrm{m}^{2} \mathrm{sr}\right)\right]$. Total quantities are derived by averaging monochromatic volumes after $\mathrm{d} \lambda$ within range $\lambda \in(0-\infty)$. The eqn. (5) is illustrated in fig. 1 .

Taking an initial assumption on the non-transparent nature of boundary surface of radiative heat transfer system, the following base equation may be written down for each point of this surface $[6,8]$

$$
L\left(\overrightarrow{\mathbf{r}}_{\mathbf{0}}, \overrightarrow{\mathbf{s}}_{\text {ref }}, \lambda\right)=\varepsilon\left(\overrightarrow{\mathbf{r}}_{\mathbf{0}}, \overrightarrow{\mathbf{s}}_{\mathbf{r e f}}, \lambda\right) L_{b b}\left(\overrightarrow{\mathbf{r}}_{\mathbf{0}}, \lambda\right)+\int_{\Omega} \rho_{k k}\left(\overrightarrow{\mathbf{r}}_{\mathbf{0}}, \overrightarrow{\mathbf{s}}_{\mathbf{r e f}}, \overrightarrow{\mathbf{s}}_{\mathbf{i n}}, \lambda\right) L_{p}\left(\overrightarrow{\mathbf{r}}_{\mathbf{0}}, \overrightarrow{\mathbf{s}}_{\mathbf{i n}}, \lambda\right) \cos \eta d \omega
$$

The equation results directly from the law of conservation of energy.

In eqn. (6) $\rho_{k k}$ is a bidirectional reflected distribution function (BRDF) describing reflexive properties of a surface, "in" refers to incident and "ref" 
reflected radiation. Angle d $\omega$ indicates an elementary solid angle, and integration is carried out on the whole hemisphere $\Omega$.

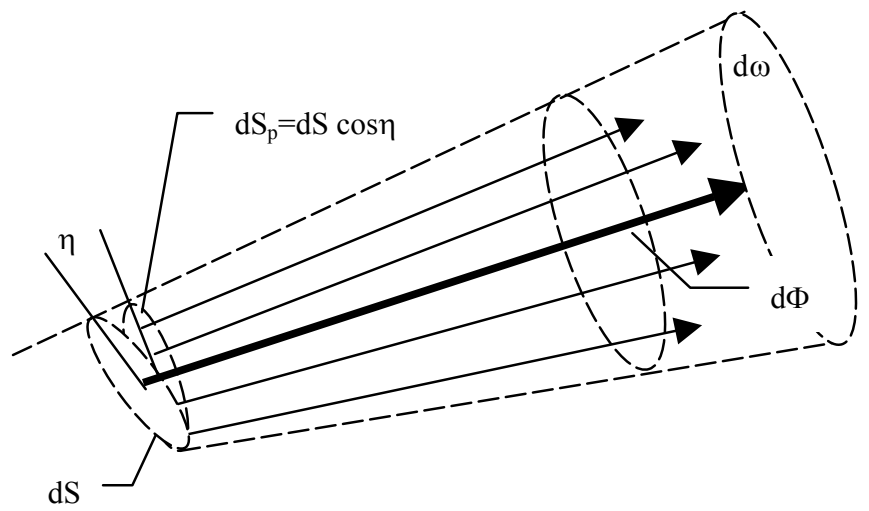

Figure 1: Definition of radiance according to eqn. (5).

Eqn. (6) means that for any point on the boundary surface $S_{i}$, whose position is determined by vector $\overrightarrow{\mathbf{r}}_{\mathbf{0}}$, radiance $L$ in the direction determined by vector $\overrightarrow{\mathbf{s}}_{\text {ref }}$ consists of the sum of primary radiation radiance (first component of eqn. (6)) and the sum of reflections in direction $\overrightarrow{\mathbf{s}}_{\text {ref }}$ of radiation incident from direction $\overrightarrow{\mathbf{s}}_{\text {in }}$ (second component - integral of eqn. (6)). Fig. 2 depicts it visually.

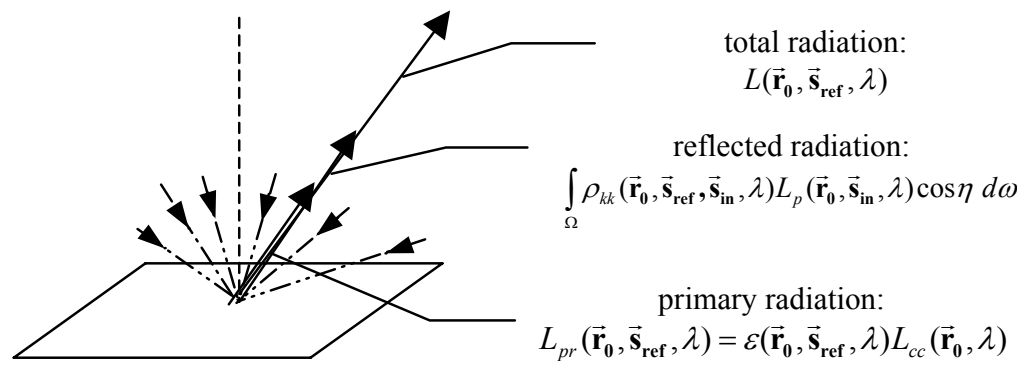

Figure 2: $\quad$ Radiance $L$ in point $\overrightarrow{\mathbf{r}}_{\mathbf{0}}$ and direction $\overrightarrow{\mathbf{s}}_{\text {ref }}$ (eqn. (6)).

The complete system of radiative heat transfer consists of numerous surfaces $S_{i}$, which most often create a closed system. For each point of every boundary surface of such a system, an integral eqn. (6) should be written. The supplement of the system of eqns (6) should be the provision of boundary conditions determining the energy-heat status of the system's boundary surfaces. These conditions are defined either as Dirichlet's or Neumann's conditions. In the former case, a function of temperature distribution on the examined surface $S_{i}$ should be specified $[3,6,8]$

$$
\underset{\substack{\mathbf{r}_{0} \in S_{i}}}{t\left(\overrightarrow{\mathbf{r}}_{\mathbf{0}}\right)}=t\left(x_{0}, y_{0}, z_{0}\right) \cdot
$$


In the latter case, surface power density $p$ proportional to the derivative of the sought function $t$, penetrating to the system from outside through boundary surface, is determined $[3,6,8]$

$$
p\left(\underset{\mathbf{r}_{0} \in S_{i}}{\overrightarrow{\mathbf{r}}_{\mathbf{0}}}\right)=p\left(x_{0}, y_{0}, z_{0}\right)=-\left.\lambda \frac{\partial t}{\partial r}\right|_{S_{i}} .
$$

In that case, as can be seen from (8), only the derivative of function $t$ is set, which means that the temperature is determined with accuracy to the constant. Thus, in order to unconditionally determine temperature distribution in the system, it is necessary to specify Dirichlet's condition for at least for one boundary surface.

A system of Fredholm's $n$ integral eqns (6) thus obtained, with boundary conditions, is practically algebraically unsolvable.

There are practically three methods of searching for a solution based on determining temperature and heat fluxes penetrating boundary surfaces. Either a series of reductions allowing to transform a system of integral eqns (6) into a system of linear equations is introduced, or a bounded system of eqns (6) is solved by using methods of numerical quadrature of integrals, or radiative heat transfer is modeled on the basis of eqns (6) thus obtaining the solution by means of simulation.

The first method, after making assumptions on radiation diffusivity and after dividing boundary surfaces into areas of finite size, leads to a system of linear equations, usually presented as follows $[6,8]$ :

$$
\sum_{j=1}^{N}\left(\frac{\delta_{i j}}{\varepsilon_{j}}-\varphi_{i j} \frac{1-\varepsilon_{j}}{\varepsilon_{j}}\right) p_{z w, j}=\sum_{j=1}^{N}\left(\delta_{i j}-\varphi_{i j}\right) \sigma T_{j}^{4} \quad \text { for } i=1,2, \ldots, N,
$$

where $N$ is the quantity of examined areas, $\varphi_{i j}$ - configuration factor of area $i^{\text {th }}$ into $\mathrm{j}^{\text {th }}$, and $\sigma$ is Stefan-Boltzman constant. Eqns (9) along with boundary conditions (7) and (8) form a uniform, determined system of linear equations, which may be solved either by classical (for small $N$ ), or iterative methods (for large $N)$.

The second method consists in making an assumption that eqn. (6) refers not only to a point but also to a certain area around it. Thanks to the use of numerical quadrature to integral equations (Newton-Cotes, Gauss and others methods) it is possible to transform them into a system of algebraic equations. These can be then solved by means of standard numerical methods.

The third method consists in modeling radiative heat transfer. Knowing the distribution of the surfaces of the system of radiative heat transfer, as well as emissive and reflexive characteristics of the surface, it is possible to model the propagation of radiation by tracing each ray starting with its emission, through reflections on boundary surfaces, and ending with the ultimate absorption. This is illustrated in fig. 3. This method can be used to trace heat powers between the system's surfaces, and later on, determine the sought temperature distributions. The classical form of the above is realized with Monte-Carlo method, which theoretically allows one to represent each heat transfer system. This kind of universality (any possible system can be modeled) constitutes an unquestionable advantage of such solutions. Unfortunately, due to the necessity to trace each 
emitted ray, and, subsequently, each reflected ray, their number increases dramatically (see fig. 3) and even state-of-the-art computers are not powerful enough to fully realize this idea.

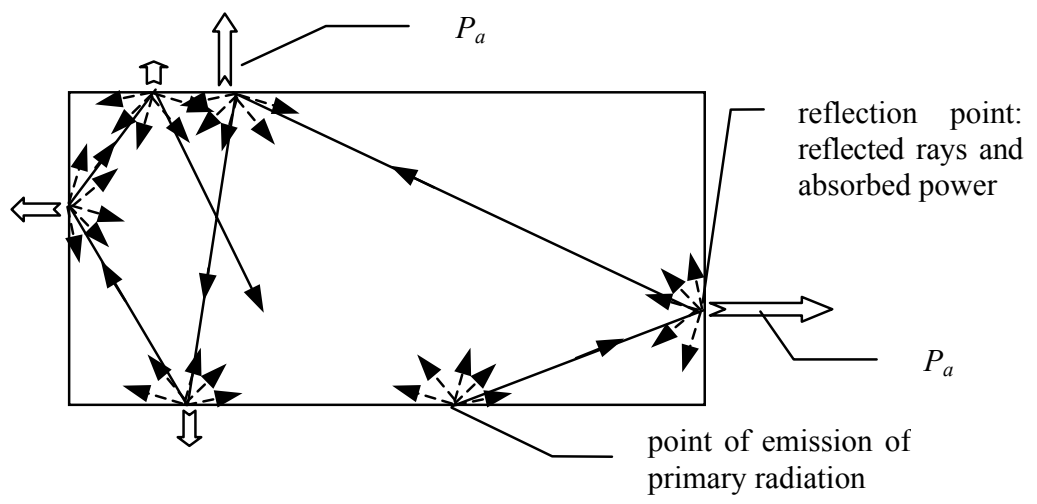

Figure 3: $\quad$ Radiation traced according to classical Monte-Carlo method.

Within this framework, research focuses on developing methods allowing one to limit the required number or rays to be examined, while retaining the required accuracy of simulation. One of such methods is backward ray tracing. The method consists in assuming rays' reverse courses, in relation to their natural courses, and examining only the ones which contribute significantly to the energy balance of selected, significant points (areas) of boundary surfaces.

\section{Computer graphics: goals and base equation}

The main goal of computer graphics software is to create a maximally realistic image of virtual reality (a so-called scene) on a monitor's screen or in the form of graphics files. This image should give the observer an impression of seeing real objects. As distinguished from many image recording technologies (photography, film, TV), computer graphics technologies do not require the recorded objects to exist in reality. It is only virtual reality that is needed, namely mathematical and physical description of objects' surfaces, and the knowledge of laws determining the generation and distribution of light (visible radiation). In computer graphics programs, the image of virtual reality is obtained by modeling and simulating, in a determined virtual space (created by boundary surfaces and light sources), of propagation of light and visual effects experienced by a virtual viewer.

For the purposes of computer graphics, Kajiya [5] introduced an equation describing the propagation of light from point $x$ in the direction of point $y$ with illumination incident on point $x$ from direction $z$. The equation was later called the visualization governing equation:

$$
L_{\lambda}(x, y)=V(x, y)\left[L_{p r, \lambda}(x, y)+\int_{\Omega} L_{i n, \lambda}(z, x) \rho_{k k, \lambda}(x, y, z) d z\right]
$$


where $\boldsymbol{L}_{\lambda}(x, y)$ is the luminance of visible radiation (light) emitted from point $x$ in the direction of point $y, V(x, y)$ - geometrical factor allowing to avoid the examination of surfaces obstructing each other in calculations, $\rho_{k k}(x, y, z)-$ see eqn. (6). Indices "pr" and "in" constitute primary emission and incident radiation.

Fig. 4 illustrates eqn. (10). It represents visually when and how an observer in point $z$ "sees" point $x$ illuminated from direction $z$.

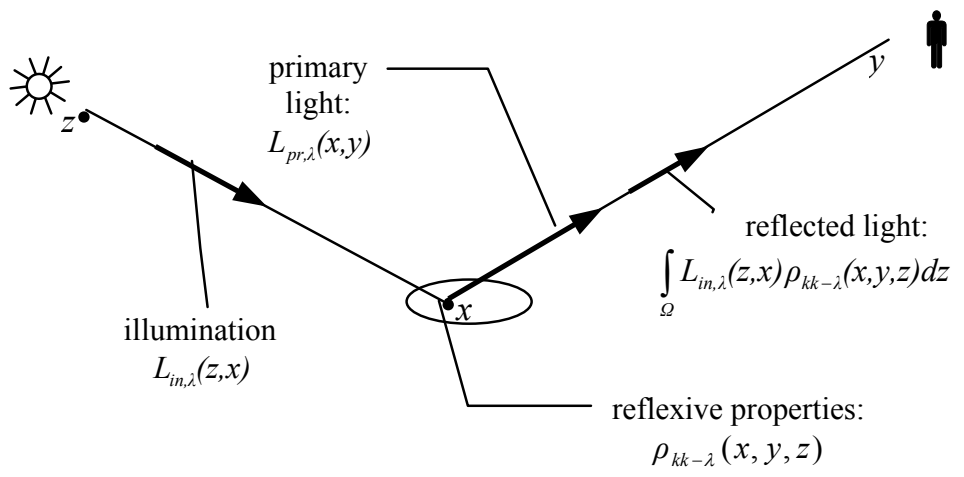

Figure 4: $\quad$ Illustration for visualization base eqn. (10).

Eqn. (10), although formulated in computer graphics terminology, contains the same physical sense as eqn. (6) expressing the law of conservation of energy for heat radiation. This can also be seen when comparing fig. 2 and 4.

\section{Computer graphics programs}

Although the goals of computer graphics programs (visualization) and of programs modeling radiative heat transfer (determining heat transfer) are different, one can see a significant concurrence of certain stages of such programs. This applies mainly to the phase of generating and tracing (history) of individual rays. This is shown in fig. 5.

Not all computer graphics programs can be adapted for heat calculations. First programs developed in late 1960s did not take into account physical phenomena related to light propagation at all, and their only purpose was to generate simple images. These programs are totally unsuitable for heat calculations.

Later programs (1970s) took into account only local illumination. The distribution of luminous flux diffused on examined surfaces and reaching the observer was specified in these programs as a function of a luminous flux incident from light sources and diffusing characteristics of observed surfaces. Surface characteristics took into consideration both diffusive and specular reflections. Eqn. (10) was the mathematical base, although interreflections were not taken into account. Instead of indirect radiation (i.e. interreflection), an artificially determined constant illumination level was introduced, whose sole purpose was to imitate effects connected with indirect illumination. In spite of these solutions, the resulting images based on models which only took into 
account direct radiation were "artificial" and considered unnatural. Due to the omission of the phenomenon of interreflection, programs based on such assumptions could not be used for modeling radiative heat transfer.

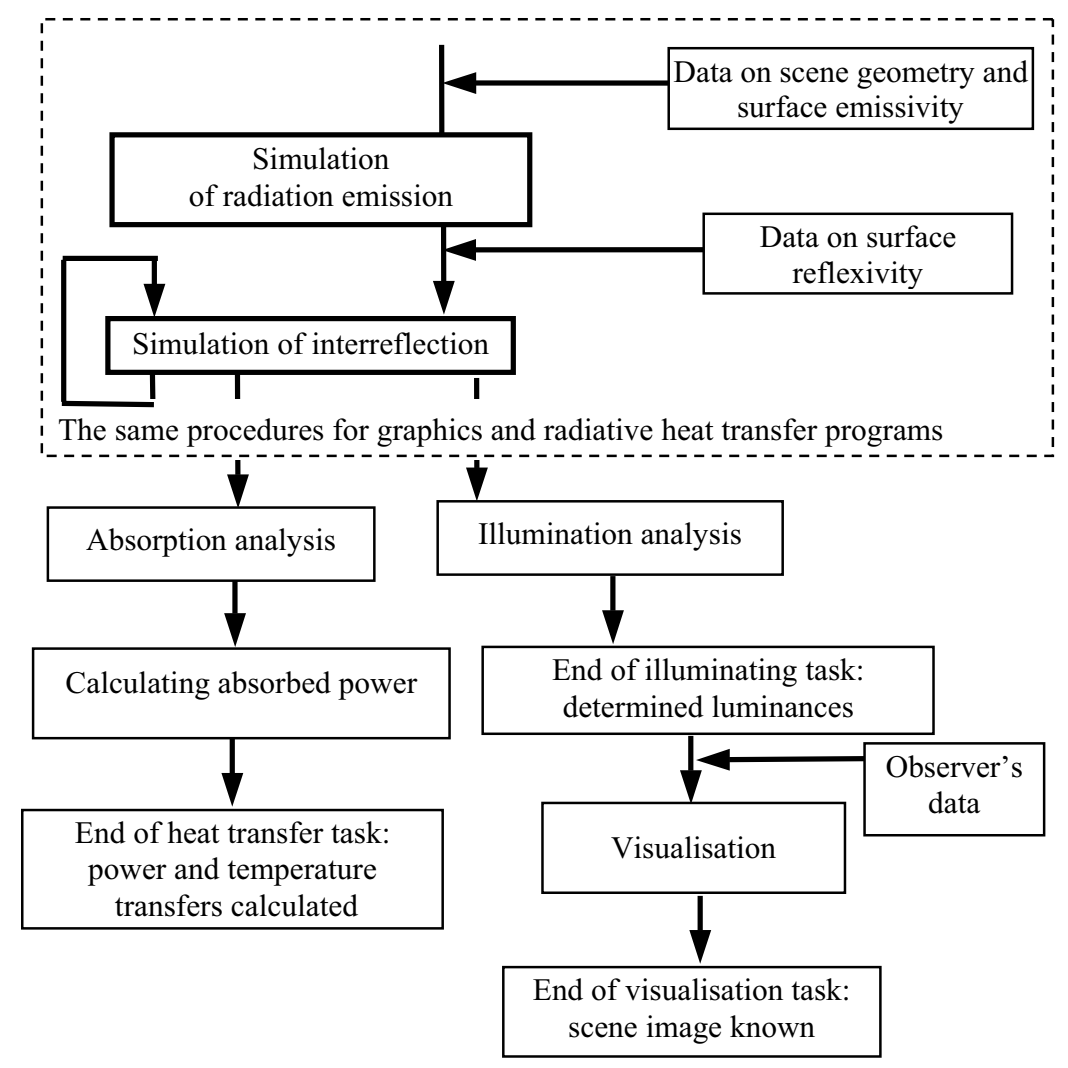

Figure 5: Functional diagram of the process of modeling radiative heat transfer and visualization.

The 1980s saw the coming of illumination programs that took into consideration both direct illumination and indirect illumination (interreflections). These are called global illumination models. Indirect lighting is caused by multiple reflections from neighboring surfaces. To take this into account, it is necessary to model the total luminous radiation distribution from the source, through interreflections and to the final absorption on a specified surface or when the observer's eye has been reached. These programs fully reflect phenomena related to generation and diffusion of light and may be used for radiative heat transfer modeling. Such programs include Radiance, Spectr System, Lightscape Visualization System, Helios and others [1, 3]. 


\section{Usability conditions}

Not every computer graphics program can be adapted for radiative heat transfer modeling. Conditions making the above possible can be divided into two groups: necessary conditions and desired conditions. The first group determines the very usability of a program for adaptation for radiative heat transfer simulation, and the second considerably facilitates this task and increases the potential accuracy of future calculations. Necessary conditions will definitely include the following:

- basing the modeling of illumination on eqn. (10),

- interreflections modeling,

- possibility to export intermediate results as files including data (but not bitmaps!) on radiance (or equivalent quantity) on determined surfaces.

Desired conditions include:

- possibility to define reflective characteristics of surfaces as a BRDF function,

- relation of a graphics program to CAD programs,

- possibility to freely shape the emissive characteristics of radiation sources,

- a graphics program is based on modeling radiant quantities, not light quantities.

\section{Transformation of heat quantities into radiant quantities}

Heat quantities (temperature, power density) are used for the description of radiative heat transfer. Computer graphics programs are based on processing light quantities (luminance) or radiant quantities (radiance). Adapting the latter to model and simulate radiative heat transfer requires heat quantities to be transformed into radiant quantities. This applies particularly to boundary conditions describing the system of radiative heat transfer.

The paper of $[3,4]$ demonstrated that boundary surface $S$ for which Dirichlet's condition (7) was determined, i.e. temperature $T=t+273$, can be replaced by energetically equivalent radiation source surface with radiance $L$ and emissivity $\varepsilon$ described by the following formula:

$$
L\left(x_{0}, y_{0}, z_{0}\right)=\frac{\sigma \varepsilon T^{4}\left(x_{0}, y_{0}, z_{0}\right)}{\pi \cos \eta}
$$

where $\sigma$ is Stefan-Boltzman constant, and angle $\eta-$ see fig. 1.

Similarly, for surfaces for which Neumann's condition (8) of equivalent surface of emissivity $\varepsilon$ was determined, radiance $L$ can be assigned [3, 4]:

$$
L\left(x_{0}, y_{0}, z_{0}\right)=\frac{p\left(x_{0}, y_{0}, z_{0}\right)+E_{\text {sum }}\left(x_{0}, y_{0}, z_{0}\right) \varepsilon}{\pi}
$$

where $E_{\text {sum }}$ is total irradiation of examined surface originating from the remaining surfaces of the system. 
Therefore, using eqns (11) and (12) the task of radiative heat transfer can be transformed into a task within the scope of light technology. Fig. 6 presents it visually.

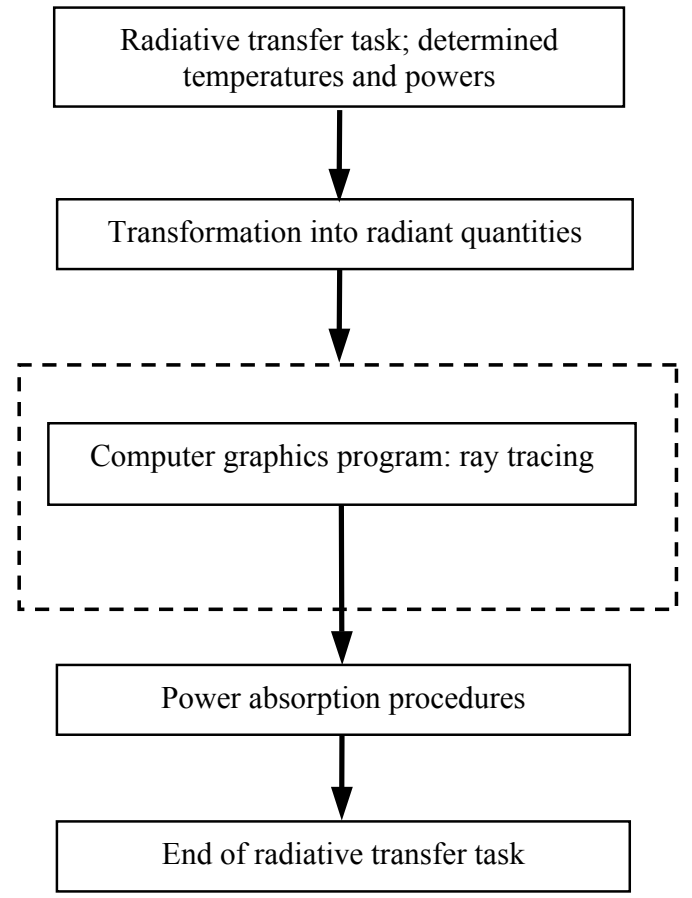

Figure 6: Stages of modeling radiative heat transfer matters with the use of computer graphics programs.

\section{Test calculations on the basis of Radiance}

As far as computer graphics is concerned, there are many program packages for scene visualization, and Radiance is one of such programs [2, 7, 9]. Utilizing the system's procedures for ray tracing and adding own, necessary procedures, a tool (program) was developed which allows to model radiative heat transfer systems. A series of calculations was done, with particular attention paid to testing simple systems for which precise analytical solutions are known. Detailed results have been provided in a series of publications [3, 4]. In all cases, a significant concurrence of simulation results (simulations, nevertheless, included a certain degree of randomness) with precise calculation results was achieved.

\section{Conclusions}

Several computer graphics programs fulfill conditions allowing them to be used not only for the simulation of illumination, but also for solving the most complex 
matters of radiative heat transfer. These programs contain procedures for modeling emission and procedures for light interreflections. Fulfillment of conditions discussed in the paper allows such programs to be used for performing the basic function of a modeling program - examining the propagation of rays in a determined system. Adding additional functions consisting in transforming heat quantities into light quantities (and vice versa) as well as functions for the calculation of power transmitted with each ray, produces a fully functional tool for modeling radiative heat transfer. Performed control calculations confirm a significant consistency of results of radiative heat transfer simulation done on the basis of Radiance package with analytical calculations.

\section{References}

[1] Ashdown I., Radiosity - A Programmer's Perspective, John Wiley \& Sons Inc, New York, 1994.

[2] Companion R., RADIANCE: A Simulation Tool for Daylight Systems, University of Cambridge, Department of Architecture, 1997.

[3] Domke K., Modelowanie symulacja i badanie radiacyjnej wymiany ciepła $w$ środowisku Radiance, ser. Rozprawy nr 378, Wyd. Pol. Pozn., Poznań, 2004, (in Polish).

[4] Domke K. \& Hauser J., Application of RADIANCE procedures for radiative heat transfer modeling: in :Computer aid design of electroheat devices ed. Hering M., Sajdak Cz. \& Wciślik M., Wyd. Pol Śląskiej, Gliwice, pp 32-49, 2002.

[5] Kajiya J., The Rendering Equations, Computer Graphics, 20(4), 1986.

[6] Modest M. F., Radiative Heat Transfer, II ed., Academic Press Amsterdam, Boston, London, N. York and Sydney, 2003.

[7] Sillon F. X. \& Puech C., Radiosity and Global Illumination, Morgan Kaufmann Publishers Inc., San Francisco and California, 1994.

[8] Siegel R. \& Howell J. R., Thermal radiation heat Transfer, Mc-Graw Hill Book Co., N. York, 1972.

[9] Ward G.L. \& Shakespeare R., Rendering with RADIANCE - The Art and Science of Lighting Visualization, Morgan Kaufmann Publ., San Francisco, 1998. 